\title{
Activation of the HLA-DRA Gene in Primary Human T Lymphocytes: Novel Usage of TATA and the $\mathrm{X}$ and $\mathrm{Y}$ Promoter Elements
}

\author{
GLENN K. MATSUSHIMA, YOSHIE ITOH-LINDSTROM, AND JENNY P.-Y. TING* \\ Lineberger Comprehensive Cancer Center and Department of Microbiology and Immunology, \\ University of North Carolina at Chapel Hill, Chapel Hill, North Carolina 27599-7295
}

Received 13 July 1992/Returned for modification 3 August 1992/Accepted 9 September 1992

\begin{abstract}
Human T lymphocytes express human leukocyte antigen (HLA)-DR-alpha (DRA) upon mitogenic or antigenic stimulation. $\mathrm{DR}^{+} \mathrm{T}$ cells are also found in a number of inflammatory and autoimmune diseases and have a proposed role in these diseases. The molecular mechanism of DR regulation in untransformed blood $T$ lymphocytes was studied here by transient transfection of DRA-chloramphenicol acetyltransferase reporter gene constructs. Several novel features of this regulation were observed. During the early stages of T-cell activation by mitogens or antigens, strong promoter induction was exhibited with the proximal 43 bp of the DRA promoter which contains a TATTA motif. Addition of upstream $X$ and $Y$ DNA elements angmented the response. This contrasts with data from transformed cell lines in which the proximal 43 bp produced no detectable promoter function, and the inclusion of $X$ and $Y$ elements is essential for basal level expression. Mutation of the TATTA motif or substitution with a functional but difierent TATA element produced errant initiation and greatly reduced gene expression. Interestingly, $T$ lymphocytes from a normal donor were $\mathbf{D R}^{+}$ prior to in vitro stimulation, and again, strong promoter activity was observed with 43 bp of proximal sequence. Unexpectedly, the presence of the $X$ and $Y$ elements correlated with a suppression of class II promoter function and surface antigen expression. This study of nontransformed lymphocytes reveals several novel features of DRA gene regulation and underscores the value and necessity of such studies.
\end{abstract}

Class II human leukocyte antigens (HLA) are central for immune responsiveness to foreign antigens. A variety of immune cells displays different patterns of class II expression. For example, B cells and dendritic cells and some macrophages express class II antigens constitutively, whereas other macrophages, microglia, astrocytes, vascular endothelium, and human $T$ cells do not normally express class II unless stimulated by antigen, cytokine, or mitogen $(3,7,14)$. The underlying molecular mechanisms which regulate the complex patterns of expression in these various immune cells are only partially understood.

A number of cis-acting and trans-acting promoter elements important for the transcriptional regulation of class II genes have been identified by using transformed cell lines as reviewed previously $(1,3,7,14,27,37)$. Most class II promoters contain three conserved proximal upstream sequences: the $W / Z$ element, $X$ element, and $Y$ element. The HLA-DR-alpha (DRA) gene also contains an additional octamer element originally found in the promoter and enhancer of immunoglobulin genes. The minimal length of the DRA promoter required for proper gene activation includes the region containing a TATA-like element as well as the $\mathrm{X}$ and Y elements collectively known as the class II box. The TATA element acts as a target sequence for the transcription factor, TFIID (TBP), which initiates the assembly of the transcription complex (15). Further upstream from the TATA, the Y element contains a reversed CCAAT box, and it forms DNA-protein complexes with specific transcription factor YB-1 (8) or NF-Y/YEBP $(16,45)$. The $X$ element is divided into the $5^{\prime} X_{1}$ and $3^{\prime} X_{2}$ sequences which interact

\footnotetext{
* Corresponding author.
}

with RF-X $(29,30)$ and hXBP-1 (20), respectively. These two sequences are essential for the activation of class II major histocompatibility (MHC) genes. Further upstream from the class II box are two additional elements: the $W$ region contains a conserved sequence known as the Servenius element (32), which can enhance gene expression by four- to fivefold, and a $\mathrm{V}$ region which acts as a cis-acting negative regulatory element (6). The importance and interdependence of these cis elements, particularly the conserved $\mathrm{X}$ and $\mathrm{Y}$ elements, are illustrated by the requirement of stereospecific and spatial alignment of these elements (42). Disruption of the distance between elements and/or their helical alignment on the DNA greatly diminishes class II gene activation.

HLA class II antigens are present on human $T$ cells activated by mitogens such as pokeweed mitogen, concanavalin A or phytohemagglutinin (PHA) $(17,44)$, alloantigens $(5,13,23)$, peptide antigens $(12,19)$, and anti-CD3 antibody which recognize the $\mathrm{T} 3$ subunit of the $\mathrm{T}$-cell receptor complex $(24,31)$. In addition, HLA class II expression is a hallmark of $\mathrm{T}$ cells isolated from patients with a number of bacterial and viral infections $(28,44)$ and autoimmune diseases such as systemic lupus erythematosus and rheumatoid arthritis $(36,44)$. In certain autoimmune diseases, the $T$ cells appear to be chronically activated; however, the activating agent has not been identified. Other proliferative stimuli of $T$ cells such as phorbol myristate acetate do not induce class II expression on T cells (24). Thus, a specific signal(s) that is distinct from the proliferative signal appears to induce the expression of HLA class II molecules on T cells.

Previous studies suggest that the TATA box, central to the formation of the preinitiation transcription complex, does not influence basic transcription of class II MHC genes (3, 43). Instead, the class II box containing $X$ and $Y$ elements is 
sufficient for proper transcriptional activity. In this report, the molecular regulation of the HLA-DRA promoter in untransformed human blood $\mathrm{T}$ cells activated by different stimuli was found to differ significantly from transformed cell lines. We provide evidence that the TATA region is critical for DRA promoter activity during $\mathrm{T}$-cell activation and augments the positive regulation provided by the $X$ and $Y$ elements of the class II box. In addition, the role of the class II box and the TATA-containing region changed during different times following T-cell stimulation. Finally, a novel role of the class II box as a negative regulatory element was observed in $\mathrm{DR}^{+} \mathrm{T}$ lymphocytes from a donor. Relief of this suppression upon mitogen stimulation was tightly associated with higher surface DR expression. These novel features of DRA gene regulation underscore the importance of studying gene regulation in primary untransformed cells.

\section{MATERIALS AND METHODS}

DRA promoter mutants. Plasmid constructs containing $-109,-53$, or -43 bp of the $5^{\prime}$ flanking sequences of the HLA-DRA gene linked to the bacterial chloramphenicol acetyltransferase (CAT) gene and the negative control (pD164-2) have been previously described (see Fig. 2A) (33, 34). Additional site-specific mutations of the DRA promoter region were made according to the procedure of Kunkel et al. (18). All mutations were made by using 5' $\Delta-152$ WT DRA-CAT as the template. It contains $152 \mathrm{bp}$ of DRA promoter sequence linked to the CAT gene cloned in a pBluescript SK ${ }^{+}$phagemid (Stratagene, La Jolla, Calif.) as previously described (22). Mutations made within the $Y$ and $X$ elements are as follows with the mutations underlined: $Y$ element mutant, Y-M1, 5'-GGAGGTTCC-3'; $X_{1}$ element mutant, X-M1, 5'-AATAGTAAT-3'; and $X_{2}$ element mutant, X-M2, 5'-ACATACA-3' as previously described (22). Mutations within the TATA region were made by using oligonucleotides containing mutations $5^{\prime}$ and $3^{\prime}$ of the TATA box and within the TATA box. The resultant mutants called TM-1 through TM-5 are shown in Fig. 4A. All mutants were transformed into DH5 $\alpha$ Escherichia coli, and mutant plasmids isolated from clones were all characterized by direct DNA sequence determination. The corresponding negative control plasmid, CAT-SK ${ }^{+}$, lacks the DRA promoter sequence.

Flow cytometric analysis. Samples of $T$ cells were labelled with primary monoclonal antibodies at predetermined dilutions. Gammapak (Genex, Gaithersburg, Md.)-purified antiCD3 (immunoglobulin G2a [IgG2a]) and anti-CD3 supernatants from ATCC CRL 8001 hybridoma were a kind gift of Olivera Finn, University of Pittsburgh. Antibodies for the B1 (IgG2a) and MY-4 (IgG2b) markers for B cells and macrophages, respectively, were purchased from Coulter Immunology (Hialeah, Fla.). Monoclonal anti-HLA-DR monomorphic (IgG2a) antibodies were produced by ATCC HB 55 (Rockville, Md.). Cells were labelled for $30 \mathrm{~min}$ at $4^{\circ} \mathrm{C}$, washed two times in phosphate-buffered saline (PBS), and subsequently labelled with a secondary antibody consisting of either fluorescein isothiocyanate conjugated to goat antimouse IgG (Organon Teknika, West Chester, Pa.) or phycoerythrin conjugated to goat anti-mouse IgG2a for $30 \mathrm{~min}$ at $4^{\circ} \mathrm{C}$. Cells were washed two times and fixed in $0.5 \%$ paraformaldehyde. Analysis of the populations was performed with a Coulter Epic 751 (Coulter Electronics, Inc., Hialeah, Fla.).

Preparation of T cells. Peripheral blood leukocytes (PBLs) from normal donors were isolated by a modified protocol described by Boyum (4). Briefly, whole blood diluted in PBS was centrifuged through histopaque 1077 (Sigma Chemical Co., St. Louis, Mo.) at 2,000 rpm $(450 \times g)$ in an IEC CRU 5000 (Needham Heights, Mass.) for $30 \mathrm{~min}$. The interface was removed and washed with PBS and centrifuged once at $1,500 \mathrm{rpm}(200 \times g)$, three times at $1,200 \mathrm{rpm}$, and once at $800 \mathrm{rpm}$ to remove the platelets. Subsequently, the cells were placed onto a Percoll gradient of 65,55 , and $46 \%$ and centrifuged to remove the monocytes (40). The cells were washed three times in PBS, resuspended in complete T-cell medium (21), and plated onto $100-\mathrm{mm}$ tissue culture plates (Falcon, Lincoln Park, N.J.). These cultures contained residual monocytes which serve as antigen-presenting cells for antigen-stimulated and mixed lymphocyte culture (MLC)-stimulated T-cell experiments. Cultures were maintained in a $7 \% \mathrm{CO}_{2}$ incubator at $37^{\circ} \mathrm{C}$ for 3 days to eliminate the B-cell population. For anti-CD3 stimulation, $\mathrm{T}$ cells were replated onto $100-\mathrm{mm}$ tissue culture plates in fresh complete T-cell medium for $2 \mathrm{~h}$ at $37^{\circ} \mathrm{C}$ to remove any residual monocytes. Nonadherent $\mathrm{T}$ cells were washed off the plates, centrifuged, and resuspended in T-cell media. Flow cytometric analysis of these isolated $T$ cells with anti-CD3 as a marker showed a greater than $98.5 \%$ enrichment of $\mathrm{CD}^{+} \mathrm{T}$ cells.

Stimulation of $T$ cells. Two-way MLC was conducted by mixing $5 \times 10^{6}$ PBLs (described above) per ml from two different donors in $75-\mathrm{cm}^{2}$ tissue culture flasks (Falcon) containing complete T-cell media. The cultures were incubated at $37^{\circ} \mathrm{C}$ in $7 \% \mathrm{CO}_{2}$ and cultured for the indicated number of days prior to transfection with plasmid DNA.

PHA was obtained from Sigma and was used at $2 \mu \mathrm{g} / \mathrm{ml}$ to stimulate PBLs cultured at $5 \times 10^{6} / \mathrm{ml}$ in $100-\mathrm{mm}$ tissue culture plates. Cells were cultured for 3 days or as indicated in Results.

Purified protein derivative (PPD) antigen made from $M y$ cobacterium tuberculosis was used at $50 \mu \mathrm{g} / \mathrm{ml}$ to stimulate PBLs. Cells were cultured at $5 \times 10^{6} / \mathrm{ml}$ in $100-\mathrm{mm}$ tissue culture plates (Corning, Corning, N.Y.) and incubated at $37^{\circ} \mathrm{C}$ for 5 days prior to transient transfection with plasmid DNA.

For anti-CD3-stimulated T cells, goat anti-mouse IgG(Fc) diluted in PBS in a fixed concentration of $5 \mu \mathrm{g} / \mathrm{ml}$ was added to 100 -mm plastic tissue culture plates for $4 \mathrm{~h}$ and subsequently washed three times prior to the addition of $1.5 \mu \mathrm{g}$ of anti-CD3 monoclonal antibody per $\mathrm{ml}$ in $0.5 \%$ bovine serum albumin. The plates were incubated for an additional $2 \mathrm{~h}$ and washed gently four times with PBS. Highly enriched $\mathrm{T}$ cells were added at $5 \times 10^{6} / \mathrm{ml}$ and cultured at the times indicated.

Transient transfection of primary lymphocytes. For MLC-, PPD-, and PHA-stimulated cultures, all cell preparations were subjected to two sequential plastic adherence to remove monocytes and sheep erythrocyte rosetting (26) to purify the $\mathrm{T}$ cells prior to electroporation. This procedure resulted in an 85 to $95 \%$ enrichment for $\mathrm{T}$ cells as analyzed by flow cytometry. The anti-CD3 cultures remained highly enriched $(>99 \%)$ for $\mathrm{T}$ cells as described earlier and did not require further purification prior to electroporation. Each DNA plasmid was added at $25 \mu \mathrm{g} / 10 \mu \mathrm{l}$ in a 300- $\mu$ l final volume of media containing $3 \times 10^{6}$ to $5 \times 10^{6}$ cells. This mixture was incubated for $10 \mathrm{~min}$ on ice and transferred to the Gene Pulser cuvette (Bio-Rad, Richmond, Calif.), which was subsequently placed in the chamber of the Bio-Rad Gene Pulser. Electroporation parameters were $260 \mathrm{~V}$ and $960 \mu \mathrm{F}$ by using the Bio-Rad capacitance extender. Cells were transferred to 12-well Costar tissue culture plates 

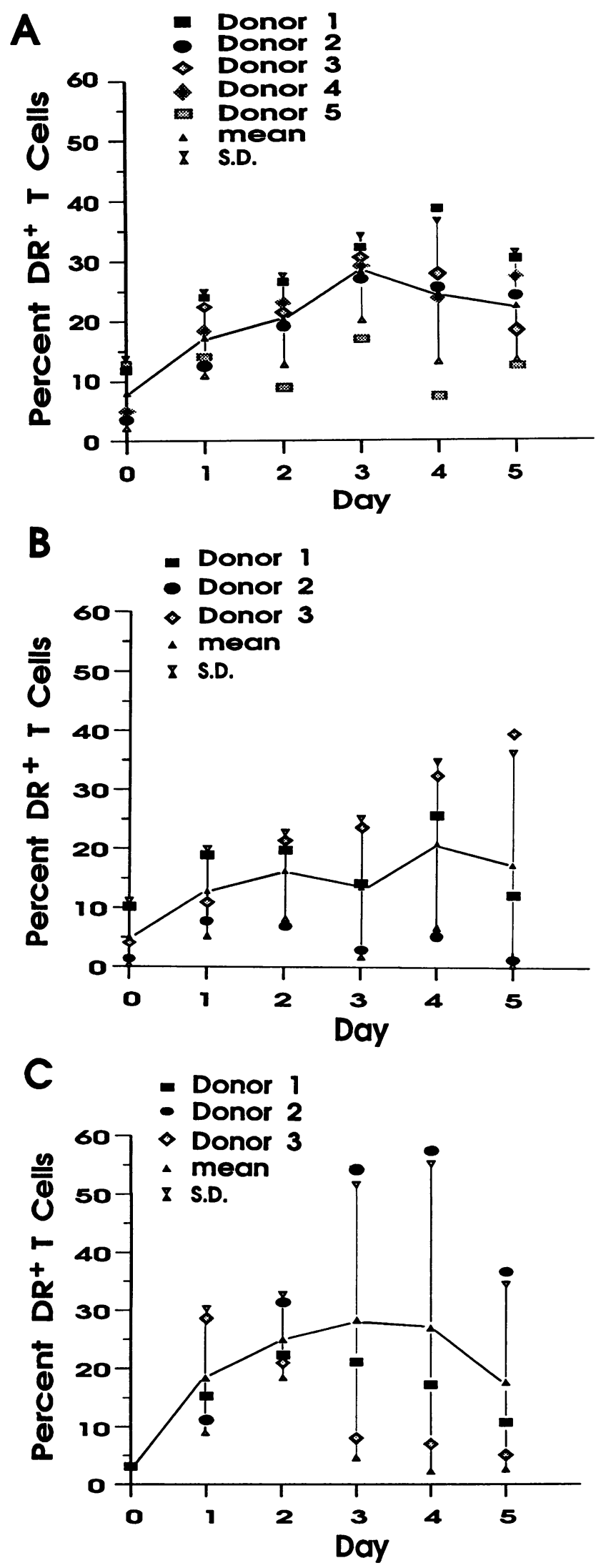

(Cambridge, Mass.) after electroporation and incubated at $37^{\circ} \mathrm{C}$ for $24 \mathrm{~h}$.

CAT assay. Assessment of CAT activity was performed as previously described $(22,33)$. Briefly, cells were harvested from tissue culture wells into 1.5-ml Eppendorf tubes, and 1 $\times 10^{6}$ to $2 \times 10^{6}$ cells were washed twice in PBS and resuspended in $25 \mu \mathrm{l}$ of $250 \mathrm{mM}$ Tris buffer (pH 7.6) and lysed by freeze-thawing in an ethanol-dry ice bath. Samples were centrifuged, and the organic extracts were assayed for CAT enzyme by using $\left[{ }^{14} \mathrm{C}\right]$ chloramphenicol (New England Nuclear, Boston, Mass.), acetyl coenzyme A buffered in 250 $\mathrm{mM}$ Tris ( $\mathrm{pH}$ 7.6). The reaction was allowed to incubate at $37^{\circ} \mathrm{C}$ from 6 to $24 \mathrm{~h}$ and terminated by the addition of ethyl acetate. The extracts were blotted onto chromagram thinlayer chromatography (Eastman Kodak, Rochester, N.Y.) and the acetylated forms of chloramphenicol were visualized by autoradiography. The thin-layer chromatography plates were quantitated for ${ }^{14} \mathrm{C}$ radioactivity by a 1214 Rackbeta counter (LKB Instruments, Gaithersburg, Md.) or by the AMBIS radioanalytic imaging system (AMBIS Systems, San Diego, Calif.). Percent acetylation was calculated as $100 \times$ (counts per minute of the acetylated forms/total counts per minute). Fold induction was calculated by dividing the percent acetylation from the DRA promoter-containing CAT plasmids by the percentage from the plasmid without DRA promoter. Statistical analysis of two values was conducted by using the Student's $t$ test.

In vitro transcription nuclear extract. Raji B-lymphoblastoid cells were used to prepare nuclear extract by using a modification of the Dignam procedure (11) as described previously (45).

In vitro transcription assays. Transcription reactions were performed as previously described $(38,45)$. Plasmids $5^{\prime} \Delta-152$ DRA-CAT and T-M1 were linearized with NcoI and were used as templates for transcription to produce a 595-bp transcript. Radiolabelled internal standard consisted of a 494-base transcript (kindly supplied by R. Kole, University of North Carolina) which was added to each reaction $(2,000$ cpm per reaction) to serve as a control for RNA recovery.

\section{RESULTS}

Cell surface HLA-DRA expression on activated primary $T$ cells. MLC, anti-CD3, and PHA stimulation have been previously shown to induce class II expression on human $\mathrm{T}$ lymphocytes. Figure 1A confirms these findings; the kinetics of class II expression on T cells stimulated by MLC shows a gradual increase over 3 days, reaching a peak at approximately day 3. A disadvantage of MLC is that matching donors is difficult and it contains a heterogeneous population of antigen-presenting cells as well as $T$ cell, $B$ cell, and other cell types responding to alloantigen. We alleviated most of these variables by stimulating $T$ cells with immobilized anti-CD3 antibody. As in MLC-stimulated T cells, $T$ cells activated by immobilized anti-CD3 antibody also induced a similar kinetics of cell surface class II antigen expression

FIG. 1. Percentage of activated human $\mathrm{T}$ cells expressing cell surface HLA-DR molecules. T cells from various normal donors were activated in either MLC (A), immobilized anti-CD3 antibody (B), or PHA (C) over a 5-day incubation period. The T cells were harvested at the indicated days and dual labelled to detect $\mathrm{DR}^{+}$ $\mathrm{CD}^{+} \mathrm{T}$ cells by flow cytometry. A minimum of three donors was analyzed for each stimulant. The curved line indicates the mean of the datum points. 

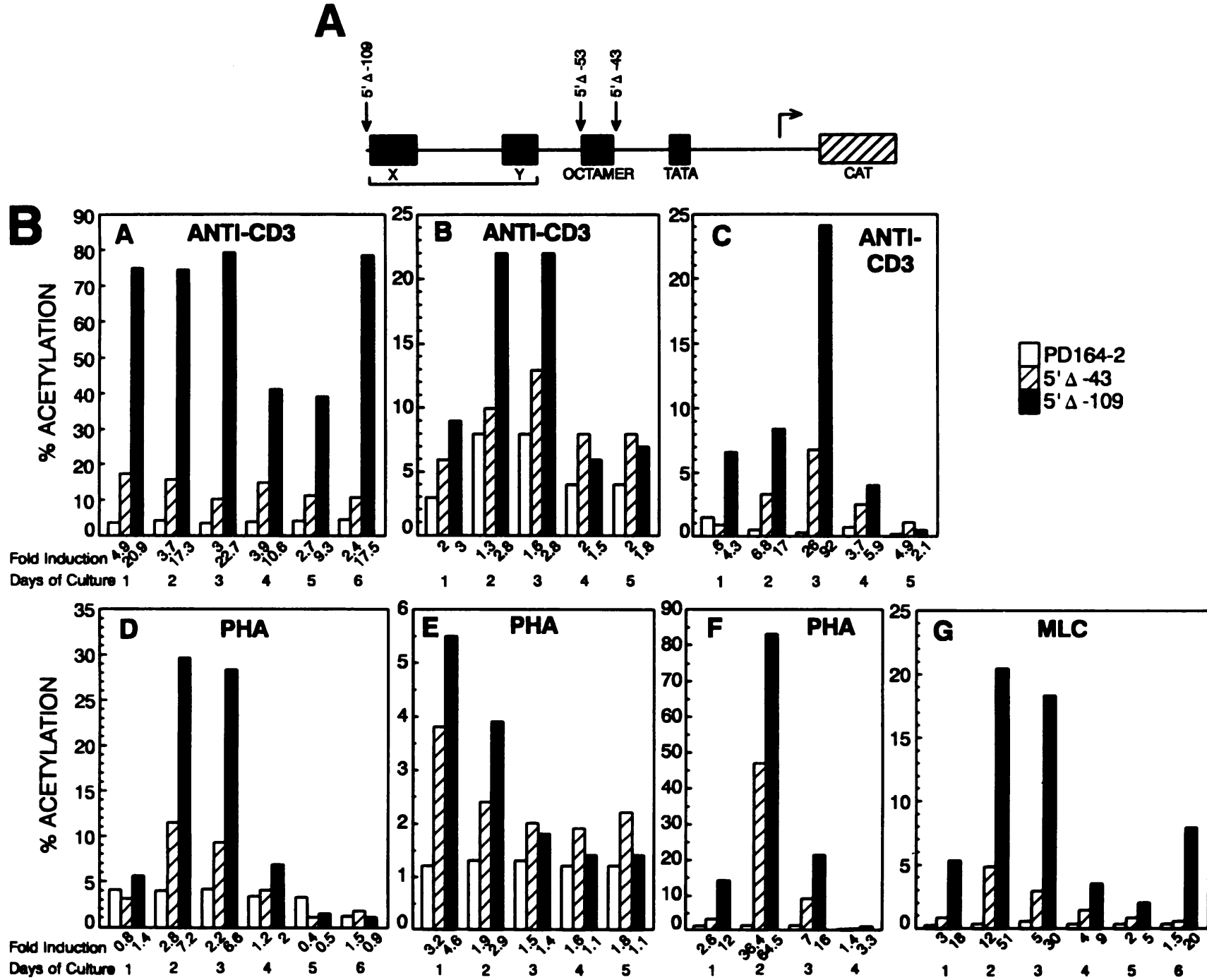

FIG. 2. Transfection of primary human T cells activated by MLC, anti-CD3, or PHA. (A) 5' $\Delta-43$ contains the DRA promoter sequences including the TATA box, $5^{\prime} \Delta-53$ further contains the octamer, and $5^{\prime} \Delta-109$ contains the $X$ and $Y$ elements. pD164-2 is a negative control containing the CAT gene but not DRA sequences. (B) CAT activity produced by primary human $\mathrm{T}$ cells transiently transfected and activated for the days indicated. Extracts of $T$ cells transfected with pD164-2, 5' $\Delta-43$, or 5' $\Delta-109$ were analyzed for CAT activity by thin-layer chromatography and quantitated by AMBIS. The histograms show relative amounts of acetylated chloramphenicol reaction products. Panels A, B, and C are anti-CD3-stimulated T cells from three different donors. Panels D, E, and F are PHA-stimulated T cells from three different donors. Panel G is a two-way MLC. Fold induction is calculated as described in the text with the negative control (pD164-2) assigned a value of 1 .

over a 5-day period, although the values are slightly lower than those for allogeneic T cells (Fig. 1B). PHA-stimulated T cells displayed a similar pattern (Fig. 1C). Unstimulated resting $T$ cells do not express significant $(<4 \%)$ HLA class II antigens (data not shown). The mean number of $\mathrm{DR}^{+} \mathrm{T}$ cells is greater in PHA stimulation than in anti-CD3 stimulation; however, analysis of the standard deviation suggests no significant difference between these two populations. Overall, a high degree of donor variability was observed, concurring with previous findings by others $(17,23)$.

Kinetic analysis of DRA promoter function in activated $T$ cells. Preliminary transfections with plasmids shown in Fig. 2A displayed different requirements for the promoter elements in alloantigen-, PPD-, or anti-CD3-stimulated $T$ cells assayed on day 6 compared with PHA-stimulated T cells assayed on day 3 (data not shown). It is critical to determine if this difference in reporter gene activity is due to different stimulatory signals, to different times of assay, or to a difference in the kinetics of $T$ cells activated by mitogen versus antigen. To distinguish between these possibilities, $T$ cells were stimulated by MLC, immobilized anti-CD3 antibody, or PHA for various intervals and transiently transfected with $5^{\prime} \Delta-43,5^{\prime} \Delta-53,5^{\prime} \Delta-109$, or the negative control pD164-2.

T cells stimulated by immobilized anti-CD3 antibody were examined first. A kinetic analysis of DRA promoter usage in anti-CD3-stimulated $T$ cells from five donors was performed, and significant donor-to-donor variations were observed as exemplified in the three experiments shown (Fig. 2B, panels A, B, and C). This is expected on the basis of other studies 
$(10,23,24)$ and our analyses of DR surface expression on $T$ lymphocytes (Fig. 1). Nonetheless, several general conclusions can be drawn from these data. Foremost, there is significant CAT expression from the $5^{\prime} \Delta-43$ construct over the negative control while inclusion of $X$ and $Y$ elements in the $5^{\prime} \Delta-109$ construct further augmented CAT expression. The level of significance for these differences was $P<0.025$. In two of three donors (panels B and C), CAT activity gradually increased upon addition of the stimulant, reaching a peak by day 3 and then subsiding by day 4 . In one donor shown in panel A, CAT activity was high on day 1 , sustained until day 3 , and then gradually decreased. On day 6, CAT expression was reactivated from the $5^{\prime} \Delta-109$ construct without an increase of CAT activity $(P<0.001)$ from the $5^{\prime} \Delta-43$ construct (Fig. 2B, panel A) or from the $5^{\prime} \Delta-53$ construct (data not shown). This pattern of reactivated CAT expression on day 6 was observed in $T$ cells from several donors, including $\mathrm{T}$ cells stimulated by alloantigens and PPD (data not shown). In an MLC (Fig. 2B, panel G), the 5' $\Delta-43$ construct expressed significant CAT activity over the negative control when the cells were activated and assayed by 1 to 4 days, and the presence of the class II box further enhanced CAT expression. Day 5 produced the lowest CAT activity for all constructs tested. However, by day 6 the cells exhibited high class II box (5' $\Delta$-109) promoter activity without significant expression from the $5^{\prime} \Delta-43$ construct (Fig. 2B, panel $G$ ) or the $5^{\prime} \Delta-53$ construct (data not shown). This pattern of promoter activity exhibited by $5^{\prime} \Delta-43$ was significantly different from the $5^{\prime} \Delta-109$ construct $(P<0.05)$ and was confirmed by using $T$ cells from two other donors which were stimulated by MLC for 6 days. These data suggest that T cells stimulated by anti-CD3 antibody (Fig. 2B, panel A) or in MLC (Fig. 2B, panel G) respond with two waves of promoter element usages and point out the dynamic nature of gene regulation in primary $\mathrm{T}$ lymphocytes.

PHA-stimulated T cells from three donors (Fig. 2B, panels D to F) showed significant levels $(P<0.025$ compared with the negative control) of CAT expression produced by $5^{\prime} \Delta-43$. $5^{\prime} \Delta-53$ also gave results identical to those of the $5^{\prime} \Delta-43$ construct (data not shown). At all early times, inclusion of the class II box substantially augmented CAT expression, as observed for the anti-CD3-stimulated $T$ cells. During the later times, a decrease in transcription occurred for both $5^{\prime} \Delta-43(P<0.025)$ and $5^{\prime} \Delta-109(P<0.001)$ without a reactivation of CAT produced by $5^{\prime} \Delta-109$ on day 6 . The diminished transcription was uniform for both $5^{\prime} \Delta-43$ and $5^{\prime} \Delta-109$. These patterns of DR promoter usage in primary $T$ cells were observed in two other donors in addition to the ones shown.

The $\mathrm{X}$ and $\mathrm{Y}$ elements of the class II box are required for promoter induction in $\mathbf{T}$ cells. Previous studies of various class II-expressing murine cells in transgenic animals indicate different requirements for $X$ or $Y$ elements (41). These studies have not been performed with any human primary cells. To assess this in human primary $T$ lymphocytes, the expression of a number of DRA promoter constructs with site-specific mutations (22) was studied. Figure 3 shows that both the $Y$ and $X$ elements are critical for class II expression in $\mathrm{T}$ cells. Mutagenesis of the $\mathrm{Y}$ or $\mathrm{X}$ element in $\mathrm{Y}$-M1 (lane 3), X-M1 (lane 4), and X-M2 (lane 5) dramatically reduced ( $P$ $<0.025)$ CAT signal compared with the positive control $5^{\prime} \Delta-152$ WT (lane 2). The negative control CAT-SK ${ }^{+}$(lane 1) did not produce a signal, as expected. This observation was consistent in five experiments using a different donor for each experiment.

The TATA box is critical for reporter gene expression in

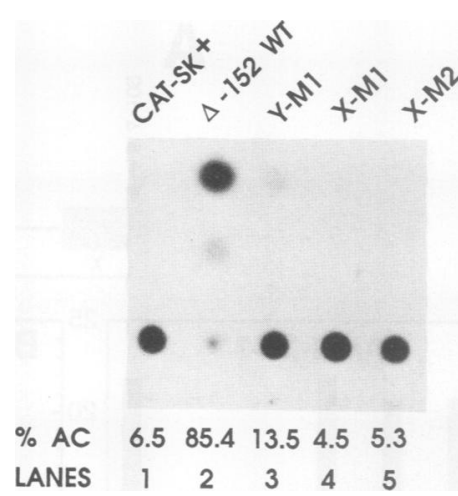

FIG. 3. Transient transfection of primary human $T$ cells with CAT reporter plasmids containing mutations within the $X$ and $Y$ elements of the DRA promoter. Freshly isolated $T$ cells were stimulated by immobilized anti-CD3 antibody and transfected on day 3. Cells were harvested $24 \mathrm{~h}$ later, and acetylated CAT products in cell extracts were separated by thin-layer chromatography and quantitated by AMBIS (\% AC). CAT-SK ${ }^{+}$is the CAT-containing negative control plasmid, $5^{\prime} \Delta-152$ WT contains 152 bp of the DRA promoter, Y-M1 contains a mutated $\mathrm{Y}$ element, $\mathrm{X}-\mathrm{M} 1$ contains a mutated $X_{1}$ element, and $X-M 2$ contains a mutated $X_{2}$ region. Similar results were obtained from five other experiments.

primary $\mathbf{T}$ lymphocytes as well as transformed cell lines. To determine the DNA sequence within $5^{\prime} \Delta-43$ that may be eliciting promoter activity, site-specific mutagenesis was employed. The most obvious motif that may be important for promoter function is TATTA at position $-24 \mathrm{bp}$, although its involvement in class II MHC promoters is controversial (see Discussion). To examine the role of the TATTA motif and juxtaposed sequences, four mutants were made as shown in Fig. 4A. In two of these mutants, T-M3 and T-M4, respectively, sequences immediate upstream and downstream of the TATTA box were mutated. In T-M1, the DRA TATTA box was replaced with TGTCA. Finally, in T-M5, the DRA TATTA sequence was substituted with the simian virus 40 (SV40) TATA box. The rationale for this is if the TATTA sequence in DRA promoter is serving the proposed function in transcription initiation, a complete random change of this sequence would obliterate gene expression. Substitution of one TATA motif for another functional TATA would permit us to decipher if DRA promoter activation is occurring through a specific TATA element present in the DRA promoter.

Transfection of TM1 to TM5 mutants into PHA-stimulated $\mathrm{T}$ lymphocytes indicates that the TATTA motif in the DRA promoter is indeed pivotal. A representative of nine experiments shown in Fig. 4B indicates that mutations of the $5^{\prime}$ and $3^{\prime}$ sequences flanking the TATA box (lanes 3 and 4, respectively) produced no reduction of CAT activity compared with the wild-type 5' $\Delta-152$ (lane 2). In fact, in approximately half of the experiments, these mutations actually enhanced CAT expression (data not shown). In stark contrast, substitution of the DRA TATTA with the SV40 TATA box dramatically reduced CAT expression (lane 5). Similarly, in a separate experiment (Fig. 4C), a radical mutation of the TATA box (T-M1) (lane 4) also greatly reduced CAT activity. These results show that the DRA TATA box is indeed important for class II promoter function in primary $T$ lymphocytes.

Because of the controversial nature of the TATA box in class II MHC promoters, we further examined the general 
A

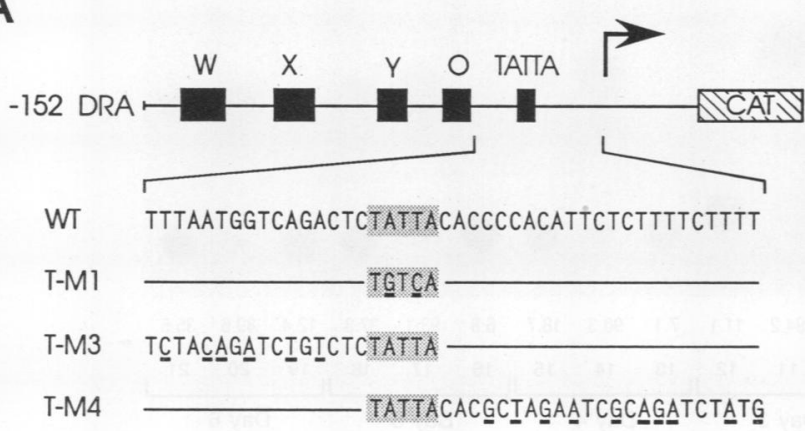

B

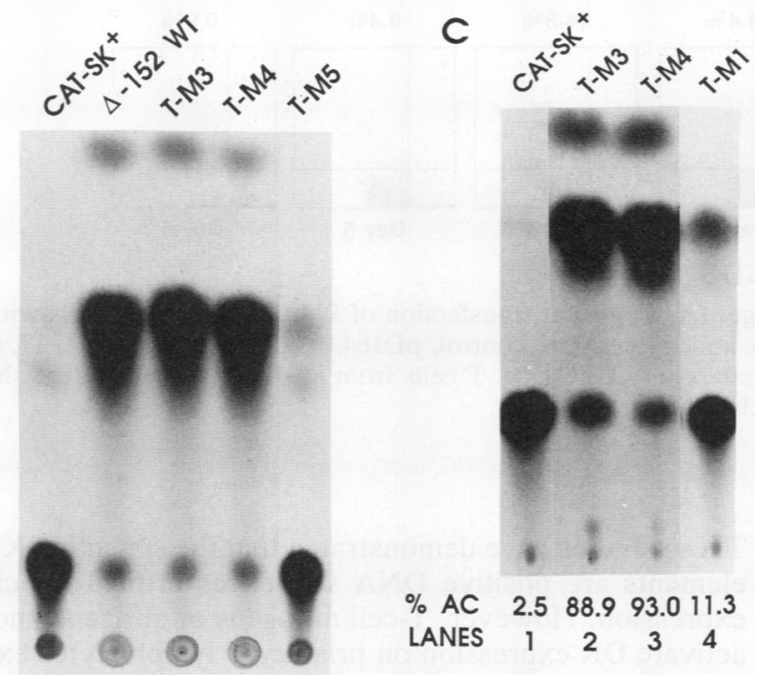

\% AC 3.194 .796 .291 .66 .8

$\begin{array}{llllll}\text { LANES } & 1 & 2 & 3 & 4 & 5\end{array}$

FIG. 4. Transient transfection of primary human $T$ cells with CAT reporter plasmids containing mutations within the TATA region of the DRA promoter. (A) Sequence of site-specific mutations within the TATA region. The TATA box is shaded. Mutated sequences are underlined. The names of these mutants are T-M1, T-M3, T-M4, and T-M5. (B) T cells activated by PHA were transfected on day 2 and assayed for CAT activity (\% AC) $24 \mathrm{~h}$ later. CAT-SK $^{+}$is the negative control, $5^{\prime} \Delta-152 \mathrm{WT}$ is the parent plasmid containing $152 \mathrm{bp}$ of the DRA promoter, T-M3 contains mutation in the 5' flanking region of TATTA, T-M4 contains mutations in the 3' flanking region of TATTA, and T-M5 contains the SV40-substituted TATTTAT box. (C) PHA-stimulated T cells transfected with the plasmid containing the mutated TATTA box. CAT-SK ${ }^{+}$is the negative control, T-M3 is the mutated $5^{\prime}$ flanking region of TATTA, T-M4 is the mutated 3' flanking region of TATTA, and T-M1 is the mutated TATA box. The level of significance for the reduction of CAT activity by T-M5 and T-M1 are $P<0.005$ and $P<0.05$, respectively.

involvement of this motif in other cells. A T-cell line which constitutively expresses DR, H9, and the B-lymphoblastoid line Raji were tested in transient transfection assays. Again, mutation of the TATA box (T-M1) obliterated gene expression as shown in Fig. 5A and B (compare lanes 2 and 3 in each experiment). To determine if this was due to aberrant initiation of transcription, in vitro transcription analysis was performed by using nuclear extract from the Raji B-lymphoblastoid cell line. As seen in Fig. 5C, three concentrations of

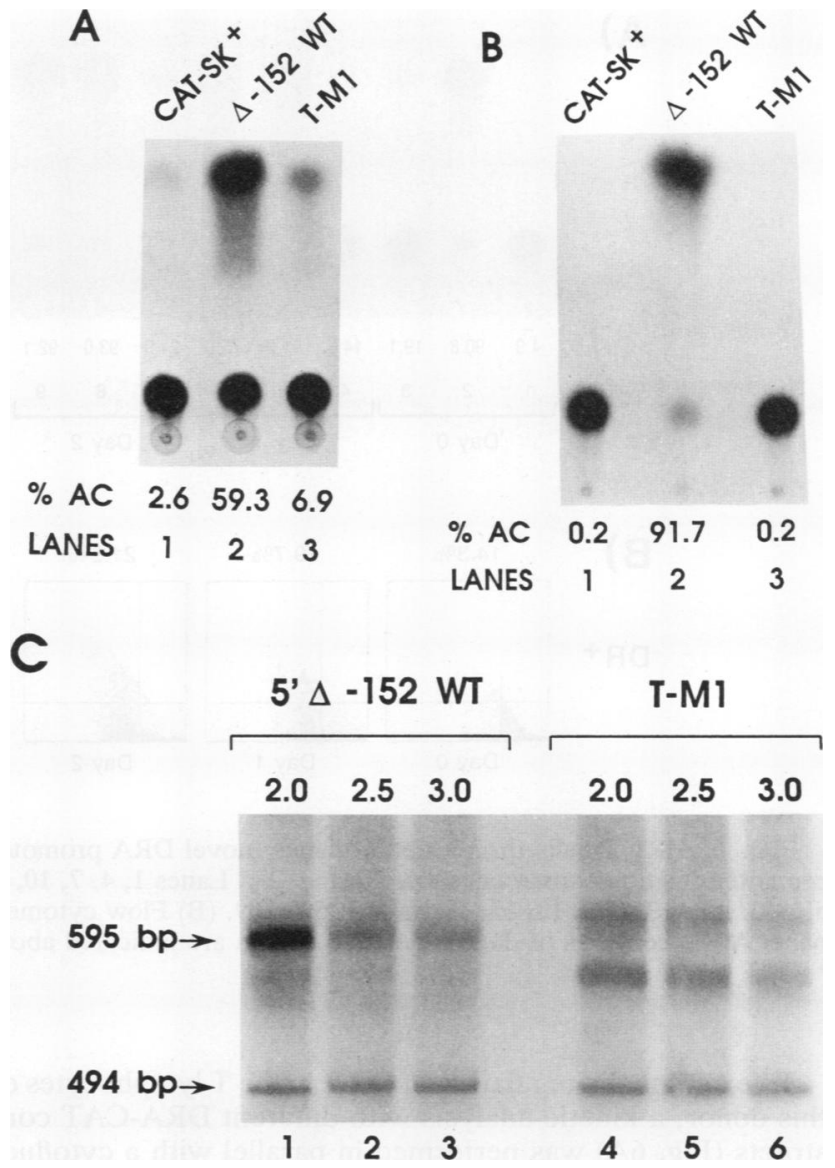

FIG. 5. Requirement of the TATA box for DRA promoter function. (A) An example from two transient transfection experiments of the T-cell line H9. CAT-SK ${ }^{+}$is the negative control; $5^{\prime} \Delta-152 \mathrm{WT}$ is the wild-type construct, and T-M1 contains the mutated TATA box. The difference between CAT activity $(\%$ AC) generated by 5 ' $\Delta-152$ and T-M1 has a $P<0.05$ value. (B) Transient transfection of Raji B cells. Lanes 1 to 3 are identical to Fig. 5A. These experiments were repeated three times. The difference between $5^{\prime} \Delta-152$ and T-M1 has a $P<0.025$ value. (C) In vitro transcription with nuclear extracts from Raji. Lanes 1, 2, and 3 show transcripts (595 bp) from the $5^{\prime} \Delta-152$ WT template at three different concentrations of DNA. Lanes 4, 5, and 6 show aberrant transcripts from the T-M1 template. A radiolabelled 494-bp transcript serves to control for RNA recovery in each reaction. An example of four in vitro transcription assays is represented.

the wild-type control (lanes 1 to 3 ) produced a transcript of the predicted size. In contrast, mutation of the TATA region resulted in the production of two aberrantly initiated transcripts. Thus, in our hands, the TATA box of the DRA promoter appears to play a conventional role in fixing the site of transcriptional initiation.

In vivo significance of DRA promoter usage: novel roles of promoter elements in a $\mathbf{D R}^{+}$donor. Resting $\mathrm{T}$ cells do not normally express class II antigens on their cell surface; however, unstimulated $\mathrm{T}$ lymphocytes from a normal donor monitored over a period of 6 months were found to consistently display elevated levels of surface DR molecules. This donor was 1 of 20 different individuals whose "normal" PBLs were analyzed in this study. In other individuals, less than $5 \%$ of the $\mathrm{T}$ cells expressed DRA molecules, and these cells exhibited no detectable DRA promoter activity as assessed by the CAT assay. 


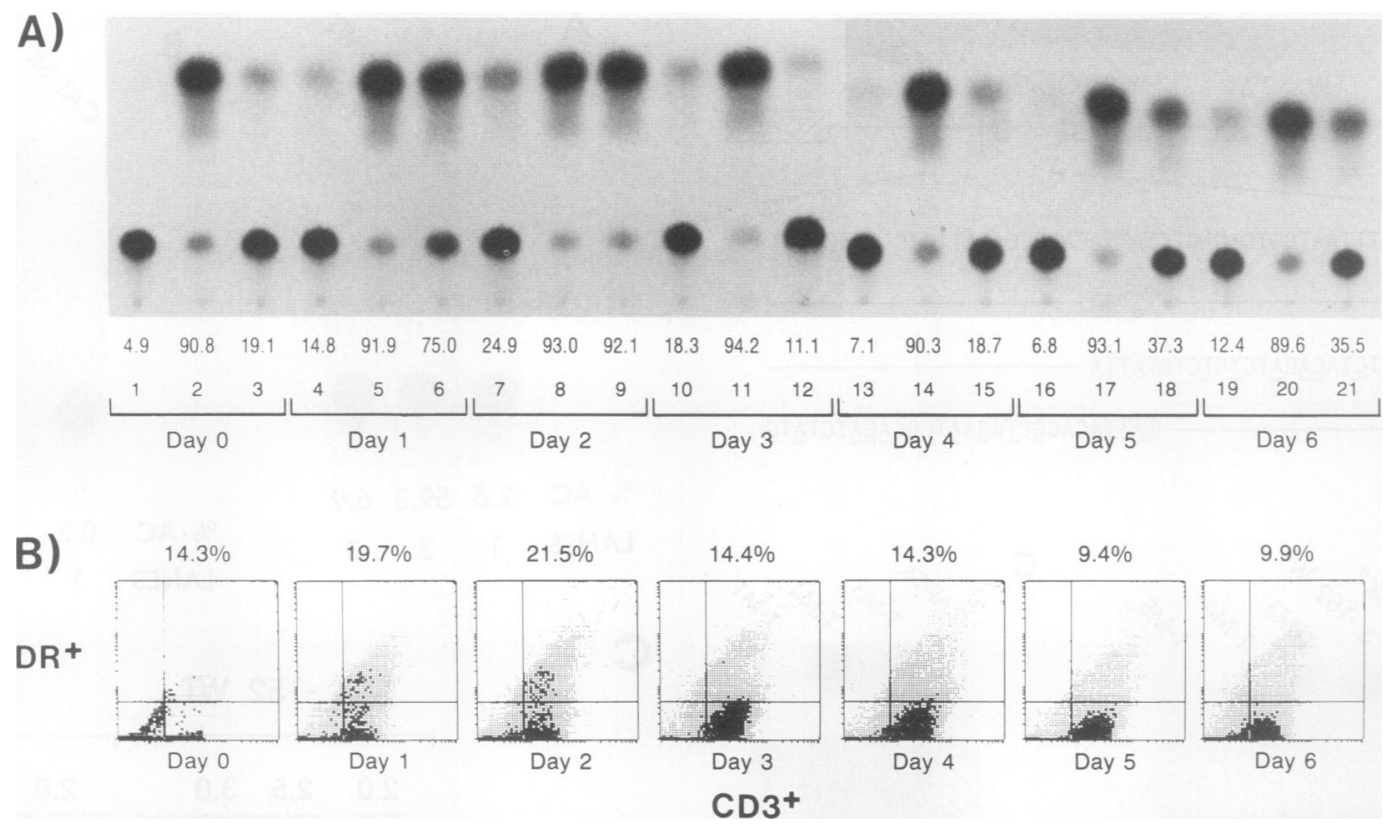

FIG. 6. $\mathrm{DR}^{+} \mathrm{T}$ cells from a donor display novel DRA promoter usage. (A) Transient transfection of PHA-stimulated T cells with DRA promoter deletion constructs shown in Fig. 2A. Lanes 1, 4, 7, 10, 13, 16, and 19, negative control, pD164-2; lanes 2, 5, 8, 11, 14, 17, and 20, $5^{\prime} \Delta$-43; lanes $3,6,9,12,15,18$, and $21,5^{\prime} \Delta-109$. (B) Flow cytometric analysis of $\mathrm{CD}^{+} \mathrm{DR}^{+} \mathrm{T}$ cells from PHA-stimulated culture shown in panel A. Percentages of double-positive $T$ cells are indicated above each panel.

To examine the regulation of DRA in the T lymphocytes of this donor, a kinetic analysis with different DRA-CAT constructs (Fig. 6A) was performed in parallel with a cytofluorometric analysis (Fig. 6B) for surface DR expression. At day $0,14.3 \%$ of the CD3-positive cells from this donor were expressing surface DR antigens (Fig. 6B, panel 1). Parallel CAT analysis at this time shows low CAT expression produced by $5^{\prime} \Delta-109$ but a surprisingly high level of CAT produced by $5^{\prime} \Delta-43$ construct (Fig. 6A, lanes 1 to 3 ). This suggests that the class II box present in $5^{\prime} \Delta-109$ is actually serving a suppressive role, resulting in $>70 \%$ suppression. Days 1 and 2 after addition of PHA, the percentage of DRA-positive cells increased to 19.7 and $21.5 \%$, respectively (Fig. 6B, panels 2 and 3). Most interesting, the suppressive effect of the class II box was also relieved at these two times as indicated by parallel CAT assay (Fig. 6A, lanes 4 to 9). The presence of the class II box resulted in a slight suppression on day 1 (compare lanes 5 and 6) which was not observed on day 2 at the height of surface DR expression (compare lanes 8 and 9). From days 3 through 5, the percentage of $\mathrm{DR}^{+}$cells dropped to $14.4 \%$ and finally to 9.4\% (Fig. 6B, panels 4 to 6). Parallel CAT analysis also shows that the suppressive effect of the class II box was regained during this time frame (Fig. 6A, lanes 10 to 18). These results reveal a novel role for the class II box and suggest that relief of this suppression can result in elevated surface DR expression. Thus, the TATA-containing region is highly active in this individual and may be down-regulated by the upstream $\mathrm{X}$ and/or $\mathrm{Y}$ element.

\section{DISCUSSION}

Previous studies of DRA gene expression have been conducted primarily with immortalized cell lines $(3,7,14)$.
These studies have demonstrated that the cis-acting $\mathrm{X}$ and $\mathrm{Y}$ elements are positive DNA sequences critical for class II expression. However, T-cell mitogens or antigens known to activate DR expression on primary $T$ lymphocytes exert no effect on DR expression by $\mathrm{DR}^{+}$or $\mathrm{DR}^{-} \mathrm{T}$-cell lines. This limitation of human $\mathrm{T}$-cell lines accentuates the importance of studies with primary $\mathrm{T}$ cells.

Our study of DRA expression in activated primary human $T$ cells has demonstrated a novel usage of a 43-bp region of the DRA promoter that contains a TATTA sequence. This 43-bp segment does not contain the $X$ and $Y$ elements but does contain the TATA box as the only recognizable element. This activity was typically more obvious during the early times of T-cell stimulation. Mutation of the TATA box and substitution with an alternate TATA sequence abrogated much of the activity, suggesting that this specific sequence is critical. Inclusion of the $\mathrm{X}$ and $\mathrm{Y}$ boxes in the DRA promoter further augmented transcriptional activity. Site specificmutagenesis shows that both the $\mathrm{X}$ and $\mathrm{Y}$ elements are important for this augmented expression in a great majority of the donor lymphocytes. In contrast, primary $\mathrm{T}$ cells from a specific donor exhibited unusual downregulation by the class II elements. These observations demonstrate that the TATA- and class II box-containing DNA regions are important components of DRA gene activation in primary $T$ lymphocytes, but the class II box may serve a negative role under some circumstances. Thus, this study reveals dynamic changes in the transcriptional control of DRA that have not been previously appreciated with transformed cells.

The TATA region is bound by the trans-acting TFIID (TBP) protein to form the transcription initiation complex and serves to guide DNA polymerase to the proper downstream start site $(3,7,14,15)$. Its role in class II MHC promoter has been disputed because linker scanning analysis in murine $\mathrm{A} \alpha$ and $\mathrm{E} \alpha$ genes by using transformed cell lines 
and gamma interferon-induced macrophages suggests that the TATA or TATA-like region is not as critical for proper initiation as the upstream X and Y elements (43). Our finding that TATA plays a predominant role in the absence of the class II box is particularly unexpected because a review also indicates that the DRA TATA is not functional (3). However, the paper cited in this review actually implied that the TATA box may be utilized for basal transcription (39), although actual mutation of the TATA box was not performed. Multiple experiments performed here utilizing sitedirected mutations of sequences immediately flanking the TATA region suggest these flanking regions do not contain identifiable positive regulatory sequences. In contrast, a complete mutation of the TATA box eliminated transcriptional activation in activated primary $T$ cells, and substitution of the DRA TATA with a SV40 TATA also diminished gene expression. A similar approach has been used to elucidate the functional dependency of adenoviral E1A on a specific TATA box $(2,35)$. Our result would suggest that different TATA boxes do not serve identical function. Taken together, these experiments strongly suggest a role for the TATA element in DRA gene expression.

Our functional analysis of the TATA box was extended to transformed cell lines by using site-specific mutants. The data unequivocally show that mutation of this element completely abrogated CAT reporter gene expression and produced aberrantly initiated transcripts in a cell-free transcription system. These experiments were performed multiple times, and the results have been striking and consistent. The following could explain the difference between our study and others. First, species-specific requirement for the TATA box could explain the difference between human and murine cells. Many TATA-less promoters exist, and some elements such as the SP-1 sequence have been shown to serve a similar role in fixing initiation sites and in interacting indirectly with the TFIID complex (15). These other class II MHC promoters likely contain these other sequences. Second, the difference in the requirement of DRA TATA motif in the two studies may be attributed to differences in the detection system or in the cells analyzed. Both the transcription initiation site and the production of an end product (CAT) were assayed in our study, while the other study did not examine the former issue. It is possible for functional CAT proteins to be produced even if the transcripts are initiating from a site other than the correct and usual site. Another explanation may be inherent differences in the cells or growth conditions of the cells. For example, in our hands the TATA-containing region exhibited moderate transcription activity during early $\mathrm{T}$-cell stimulation, but not at a delayed time.

The class II $\mathrm{X}$ and/or $\mathrm{Y}$ promoter element is critical for constitutive expression of class II antigen in B-cell lines, in gamma interferon-induced cells, and in class II positive immune cells in transgenic mice. Our 5' $\Delta-109$ constructs also showed the importance of $\mathrm{X}$ and $\mathrm{Y}$ elements for induced expression in human primary $\mathrm{T}$ cells. For the majority of donors tested, $T$ cells appear to require both the $X$ and $Y$ boxes. This is similar to that observed with $B$ cells from transgenic mice. However, in transgenic mice a major proportion of $\mathrm{B}$ cells requires the $\mathrm{Y}$ box and a minor population requires the $\mathrm{X}$ box for class II gene expression. In contrast, thymic epithelial cells only require the $X$ box for proper transcription, whereas macrophages rely solely on the $Y$ element (41). We are currently separating $T$ cells into subpopulations to determine if the exclusive usage of either the $\mathrm{X}$ box or the $\mathrm{Y}$ box exists in subpopulations of $\mathrm{T}$ cells.
Another point of investigation is the DNA-binding proteins that are specific for the class II elements. A number of DNA-binding proteins have been shown to bind these two elements, including NF-Y/YEBP and YB-1 specific for the $Y$ box, and RF-X, NF-X, and hXBP-1 specific for the $X$ box. It is of interest to determine if these DNA-binding proteins are altered in expression and/or function during T-cell activation. In contrast to the class II elements, the octamer element is unique to DRA and appears to have little influence on promoter induction during the early phase of $\mathrm{T}$-cell activation. A $5^{\prime} \Delta-53$ deletion construct which contains octamer and TATA boxes showed identical induction of CAT activity as the $5^{\prime} \Delta-43$ deletion construct containing TATA only (data not shown).

T-cell lines maintained long term in vitro by constant antigen stimulation and interleukin-2 were not used in this study, although many express HLA class II antigens (10, $25)$. However, they may not reflect as closely the in vivo physiologic situation as freshly isolated $T$ cells. This is underscored by our kinetic study which reveals shifts in promoter usage in activated $T$ cells depending on the time of assay. All $\mathrm{T}$ cells stimulated by alloantigens, peptide antigens, anti-CD3, or PHA showed predominant usage of the TATA-containing region during the first period of stimulation. In each case, the inclusion of the $X$ and $Y$ elements augmented the transcriptional activity. However, an augmentation of the class II box also was observed in allogeneic or anti-CD3-stimulated $T$ cells at a late time (day 6 ) with a diminished effect of the TATA-containing region. This reveals dynamic changes in the transcriptional control of DRA that have not been previously appreciated with transformed cells.

Interestingly, a specific donor with a higher percentage of $\mathrm{DR}^{+} \mathrm{T}$ cells showed strong CAT expression with $5^{\prime} \Delta-43$ construct prior to any stimulation. The presence of the class II box actually produced a dramatic suppression of CAT expression at day 0 . Analysis of this donor's lymphocytes 24 and $48 \mathrm{~h}$ after mitogen stimulation showed a relief from the suppressive effect of the class II box. Most important, this is correlated with an actual enhancement of $\mathrm{DR}^{+} \mathrm{T}$ cells. At later times, the percentage of $\mathrm{DR}^{+} \mathrm{T}$ cells returned to prestimulated levels, and the suppressive role of the class II box was again observed. Reanalysis of this kinetic study using this donor has not been possible because of insufficient numbers of cells and infrequent availability of these donors; however, a similar observation was made for two other donors. These results suggest the involvement of a negative factor which may bind or interact with either or both of the $\mathrm{X}$ and $\mathrm{Y}$ elements to control DRA gene expression prior to mitogen stimulation. A candidate for such a negative regulator is YB-1, which binds the Y box of the DRA promoter. YB-1 has been shown to downregulate DRA-CAT expression and subsequently decrease class II MHC mRNA and cell surface antigen expression (9). Such a mechanism may also be critical in primary $\mathrm{T}$ cells normally silenced for class II expression.

In summary, the molecular analysis of activated $\mathrm{DR}^{+} \mathrm{T}$ lymphocytes has revealed several novel features of gene regulation. Some of these features are dramatically different from that observed with transformed cells and underscore the significance of such studies despite technical and logistic difficulties of these analyses. The dynamic nature of these pathways is likely to reveal mechanisms of general and broad relevance to $\mathrm{T}$-cell activation. 


\section{ACKNOWLEDGMENTS}

We thank A. Yurochko and S. Haskill for providing us with PBLs from healthy donors, S. Hand and $\mathrm{O}$. Finn for providing valuable reagents, J. Smith for flow cytometric analysis, and $M$. Davis for excellent secretarial assistance. We are also indebted to the technical contributions provided by T. Moore and $\mathrm{H}$. Moses.

This work was supported in part by National Cancer Institute grant CA48185, an American Cancer Society Faculty Award, and a Jefferson Pilot Award to J.P.-Y.T.; by a National Institutes of Health training grant and an Arthritis Foundation Postdoctoral Fellowship to G.K.M.; and by an NIH training grant and a National Institutes of Health individual postdoctoral fellowship to Y.I.-L.

\section{REFERENCES}

1. Accolla, R. S., P. Dellabona, L. Scarpellino, G. Cassa, and S. Sartoris. 1990. A family of trans-acting factors with distinct regulatory functions control expression of MHC class II genes. Immunol. Res. 9:20-33.

2. Babiss, L. E., and L. D. Vales. 1991. Promoter of the adenovirus polypeptide IX gene: similarity to E1B and inactivation by substitution of the simian virus 40 TATA elements. J. Virol. 65:598-605.

3. Benoist, C., and D. Mathis. 1990. Regulation of major histocompatibility complex class II genes: X, Y and other letters of the alphabet. Annu. Rev. Immunol. 8:681-715.

4. Boyum, A. 1968. Isolation of mononuclear cells and granulocytes from human blood. Scand. J. Clin. Lab. Invest. 21(Suppl. 97):77-89.

5. Charron, D. J., E. G. Engleman, C. J. Benike, and H. O. McDevitt. 1980. Ia antigens on alloreactive $T$ cells in man detected by monoclonal antibody. J. Exp. Med. 152:127s-136s.

6. Cogswell, J. P., P. V. Basta, and J. P.-Y. Ting. 1990. X-box binding proteins positively and negatively regulate transcription of the HLA-DRA gene through interaction with discrete upstream W and V elements. Proc. Natl. Acad. Sci. USA 87:7703 7707.

7. Cogswell, J. P., N. Zeleznik-Le, and J. P.-Y. Ting. 1991. Transcriptional regulation of the HLA-DRA gene. Crit. Rev. Immunol. 11:87-112.

8. Didier, D. K., J. Schiffenbauer, S. L. Woulfe, M. Zacheis, and B. D. Schwartz. 1988. Characterization of the cDNA encoding a protein binding to the major histocompatibility complex class II Y box. Proc. Natl. Acad. Sci. USA 85:7322-7326.

9. Didier, D. K., S. L. Woulfe, and B. D. Schwartz. 1990. Characterization of a DNA-binding protein system which is inversely correlated with class II expression. Immunol. Res. 9:69-76.

10. Diedrichs, M., and D. J. Schendel. 1989. Differential surface expression of class II isotypes on activated CD4 and CD8 cells correlates with levels of locus-specific mRNA. J. Immunol. 142:3275-3280.

11. Dignam, J. D., R. M. Lebovitz, and R. G. Roeder. 1983. Accurate transcription initiation by RNA polymerase II in a soluble extract from isolated mammalian nuclei. Nucleic Acids Res. 11:1475-1489.

12. Ferrari, C., P. Massimo, A. Penna, A. Bertoletti, A. Valli, A. Cavalli, G. Pasetti, and F. Fiaccadori. 1992. Autopresentation of hepatitis B virus envelope antigen by T cells. J. Virol. 66:25362540.

13. Fu, S. M., N. Chiorazzi, C. Y. Wang, G. Montazeri, H. G. Kunkel, H. S. Ko, and A. B. Gottlieb. 1978. Ia-bearing lymphocytes in man. J. Exp. Med. 148:1423-1428.

14. Glimcher, L. H., and C. J. Kara. 1992. Sequences and factors: a guide to MHC class II transcription. Annu. Rev. Immunol. 10:13-50.

15. Greenblatt, J. 1991. Roles of TFIID in transcriptional initiation by RNA polymerase II. Cell 66:1067-1070.

16. Hooft van Huijsduijenen, R. A. M., J. Bollekens, A. Dorn, C. Benoist, and D. Mathis. 1987. Properties of a CCAAT boxbinding protein. Nucleic Acids Res. 15:7265-7282.

17. Ko, H.-S., S. M. Fu, R. J. Winchester, D. T. Y. Yu, and H. G. Kunkel. 1979. Ia determinants on stimulated human T lympho- cytes. J. Exp. Med. 150:246-255.

18. Kunkel, T. A., J. D. Roberts, and R. A. Zakour. 1987. Rapid and efficient site-specific mutagenesis without phenotypic selection. Methods Enzymol. 154:367-382.

19. Lanzavecchia, A., E. Roosnek, T. Gregory, P. Berman, and S. Abrignani. 1988. T cells can present antigens such as gp 120 targeted to their own surface molecules. Nature (London) 334:530-532.

20. Liou, H.-C., M. R. Boothby, P. W. Finn, R. Davidon, N. Nabavi, N. Zeleznik-Le, J. P.-Y. Ting, and L. H. Glimcher. 1990. A new member of the leucine zipper class of proteins that binds to HLA-DRA promoter. Science 247:1581-1584.

21. Matsushima, G. K., and S. A. Stohlman. 1991. Distinct subsets of accessory cells activate Thy- $1^{+}$triple negative $\left(\mathrm{CD}^{-}, \mathrm{CD}^{-}\right.$ $\mathrm{CD}^{-}$) cells and Th-1 delayed-type hypersensitivity effector $\mathrm{T}$ cells. J. Immunol. 146:3322-3331.

22. Moses, H., R. B. Panek, E. N. Benveniste, and J. P.-Y. Ting. 1992. Usage of primary cells to delineate IFN- $\gamma$-responsive DNA elements in the HLA-DRA promoter and to identify a novel IFN- $\gamma$-enhanced nuclear factor. J. Immunol. 148:36433651

23. Oshima, S., and D. D. Eckels. 1990 . Selective expression of class II MHC isotypes by MLC-activated human T lymphocytes. Hum. Immunol. 27:208-219.

24. Oshima, S., and D. D. Eckels. 1990. Selective signal transduction through the $\mathrm{CD} 3$ or $\mathrm{CD} 2$ complex is required for class II MHC expression by human T cells. J. Immunol. 145:4018-4025.

25. Pawelec, G., and H. J. Buhring. 1990. Expression of MHC class II epitopes on human $\mathrm{T}$ lymphocyte clones. Cell. Immunol. 127:520-526.

26. Pellegrino, M. A., S. Ferrone, M. P. Dietrich, and R. A. Reisfeld. 1975. Enhancement of sheep red blood cell human lymphocyte rosette formation by sulfhydryl compound 2-amino ethylisothiouronium bromide. Clin. Immunol. Immunopathol. 3:324-333.

27. Peterlin, B. M., G. Andersson, E. Lotscher, and S. Tsang. 1990. Transcriptional regulation of HLA class II genes. Immunol. Res. 9:164-177.

28. Popovic, M., G. Lange-Wantzin, P. S. Sarin, D. Mann, and R. C. Gallo. 1983. Transformation of human umbilical cord blood $\mathrm{T}$ cells by human T-cell leukemia/lymphoma virus. Proc. Natl. Acad. Sci. USA 80:5402-5406.

29. Reith, W., E. Barras, S. Satola, M. Kobr, D. Reinhart, C. Herrero-Sanchez, and B. Mach. 1989. Cloning of the major histocompatibility complex class II promoter binding protein affected in a hereditary defect in class II gene regulation. Proc. Natl. Acad. Sci. USA 86:4200-4204.

30. Reith, W., C. Herrero-Sanchex, M. Kobr, P. Silacci, C. Berte, E. Barras, S. Fey, and B. Mach. 1990. MHC class II regulatory factor RF-X has a novel DNA-binding domain and a functionally independent dimerization domain. Genes Dev. 4:1528-1540.

31. Robbins, P. A., V. C. Maino, N. L. Warner, and F. M. Brodsky. 1988. Activated $T$ cells and monocytes have characteristic patterns of class II antigen expression. J. Immunol. 141:12811287.

32. Servenius, B., L. Rask, and P. A. Peterson. 1987. Class II genes of the human major histocompatibility complex. J. Biol. Chem. 262:8759-8766.

33. Sherman, P. A., P. V. Basta, T. L. Moore, A. M. Brown, and J. P.-Y. Ting. 1989. Class II box concensus sequences in the HLA-DRA gene: transcriptional function and interaction with nuclear proteins. Mol. Cell. Biol. 9:50-56.

34. Sherman, P. A., P. V. Basta, and J. P.-Y. Ting. 1987. Upstream DNA sequences required for tissue-specific expression of the HLA-DRA gene. Proc. Natl. Acad. Sci. USA 84:4254-4258.

35. Simon, M. C., R. J. Rooney, T. M. Fisch, N. Heintz, and J. R. Nevins. 1990. E1A-dependent transactivation of the $c$-fos promoter requires the TATAA sequence. Proc. Natl. Acad. Sci. USA 87:513-517.

36. Smith, M. D., and P. J. Roberts-Thomson. 1990. Lymphocyte surface marker expression in rheumatic diseases: evidence for prior activation of lymphocytes in vivo. Ann. Rheum. Dis. 49:81-87.

37. Sullivan, K. E., A. F. Calman, M. Nakanishi, S. Y. Tsang, Y. 
Wang, and B. M. Peterlin. 1987. A model for transcriptional regulation of MHC class II genes. Immunol. Today 8:289-293.

38. Swick, A. G., M. C. Blake, J. W. Kahn, and J. C. Axizkhan. 1989. Functional analysis of GC element binding and transcription in the hamster dihydrofolate reductase gene promoter. Nucleic Acids Res. 17:9291-9304.

39. Tsang, S. Y., M. Nakanishi, and B. M. Peterlin. 1990. Mutational analysis of the DRA promoter: cis-acting sequences and transacting factors. Mol. Cell. Biol. 10:711-719.

40. Ulmer, A. J., and H.-D. Flad. 1979. Discontinuous density gradient separation of human mononuclear leukocytes using percoll as gradient medium. J. Immunol. Methods 30:1-10.

41. van Ewijk, W., Y. Ron, J. Monaco, J. Kappler, P. Marrack, M. Le Meur, P. Gerlinger, B. Durand, C. Benoist, and D. Mathis. 1988. Compartmentalization of MHC class II gene expression in transgenic mice. Cell 53:357-370.
42. Vilen, B. J., J. P. Cogswell, and J. P.-Y. Ting. 1991. Stereospecific alignment of the $\mathrm{X}$ and $\mathrm{Y}$ elements is required for major histocompatibility complex class II DRA promoter function. Mol. Cell. Biol. 11:2406-2415.

43. Viville, S., V. Jongeneel, W. Koch, R. Mantovani, C. Benoist, and D. Mathis. 1991. The E $\alpha$ promoter: a linker scanning analysis. J. Immunol. 146:3211-3217.

44. Yu, D. T. Y., J. M. McCune, S. M. Fu, R. J. Winchester, and H. G. Kunkel. 1980. Two types of Ia-positive T cells. J. Exp. Med. 152:89s-98s.

45. Zeleznik-Le, N. J., J. C. Azizkhan, and J. P.-Y. Ting. 1991. An affinity-purified serum inducible CCAAT box-binding protein (YEBP) functionally regulates the expression of a human class II major histocompatibility gene and the herpes simplex virus thymidine kinase gene. Proc. Natl. Acad. Sci. USA 88:18731877. 